\title{
Involvement of glutathione and glutathione metabolizing enzymes in human colorectal cancer cell lines and tissues
}

\author{
AREUM DASEUL KIM ${ }^{1}$, RUI ZHANG ${ }^{1,2}$, XIA HAN $^{1}$, KYOUNG AH KANG $^{1}$, MEI JING PIAO ${ }^{1}$, \\ YOUNG HEE MAENG ${ }^{1}$, WEON YOUNG CHANG ${ }^{1}$ and JIN WON HYUN ${ }^{1}$ \\ ${ }^{1}$ Department of Biochemistry, School of Medicine and Institute for Nuclear Science and Technology, \\ Jeju National University, Jeju 690-756, Republic of Korea; ${ }^{2}$ School of Medical Science and Laboratory Medicine, \\ Jiangsu University, Zhenjiang, Jiangsu 212013, P.R. China
}

Received February 21, 2014; Accepted April 30, 2015

DOI: $10.3892 / \mathrm{mmr} .2015 .3902$

\begin{abstract}
Reduced glutathione (GSH) is an abundant tripeptide present in the majority of cell types. GSH is highly reactive and is often conjugated to other molecules, via its sulfhydryl moiety. GSH is synthesized from glutamic acid, cysteine, and glycine via two sequential ATP-consuming steps, which are catalyzed by glutamate cysteine ligase (GCL) and GSH synthetase (GSS). However, the role of GSH in cancer remains to be elucidated. The present study aimed to determine the levels of GSH and GSH synthetic enzymes in human colorectal cancer. The mRNA and protein expression levels of GSH, the catalytic subunit of GCL (GCLC) and GSS were significantly higher in the following five colon cancer cell lines: Caco-2, SNU-407, SNU-1033, HCT-116, and HT-29, as compared with the normal colon cell line, FHC. Similarly, in 9 out of 15 patients with colon cancer, GSH expression levels were higher in tumor tissue, as compared with adjacent normal tissue. In addition, the protein expression levels of GCLC and GSS were higher in the tumor tissue of 8 out of 15 , and 10 out of 15 patients with colon cancer respectively, as compared with adjacent normal tissue. Immunohistochemical analyses confirmed that GCLC and GSS were expressed at higher levels in colon cancer tissue, as compared with normal mucosa. Since GSH and GSH metabolizing enzymes are present at elevated levels in colonic tumors, they may serve as clinically useful biomarkers of colon cancer, and/or targets for anti-colon cancer drugs.
\end{abstract}

Correspondence to: Professor Jin Won Hyun, Department of Biochemistry, School of Medicine and Institute for Nuclear Science and Technology, Jeju National University, 311 Jejudaehak-ro, Jeju 690-756, Republic of Korea

E-mail: jinwonh@jejunu.ac.kr

Key words: reduced glutathione, human colorectal cancers, glutamate cysteine ligase, glutathione synthetase

\section{Introduction}

Reduced glutathione (GSH) is an abundantly expressed intracellular thiol peptide in aerobic cells, which is known to be involved in cellular detoxification, antioxidant defense, maintenance of thiol status, and modulation of cellular proliferation $(1,2)$. The concentration of intracellular GSH is influenced by numerous factors, and reflects the balance between rates of consumption and de novo synthesis. The synthesis of GSH proceeds via two ATP-dependent enzymatic steps: Formation of $\gamma$-glutamylcysteine from glutamate and cysteine, which is followed by formation of GSH from $\gamma$-glutamylcysteine and glycine. The initial step of GSH biosynthesis is rate-limiting and is catalyzed by glutamate cysteine ligase (GCL), a heterodimer that consists of a $73 \mathrm{kDa}$ catalytic subunit (GCLC) and a $29 \mathrm{kDa}$ modulatory subunit (3). The second step of GSH synthesis is catalyzed by glutathione synthetase (GSS), which is a $52 \mathrm{kDa}$ homodimer. GSH metabolism has a complex role in both cancer and anticancer therapy (4). Furthermore, GSH is important in the detoxification of carcinogens, and elevated levels of GSH may increase the resistance of numerous types of tumor to chemotherapy and radiotherapy (5-7).

Various factors are associated with the initiation and development of cancer, including genetic, environmental and dietary influences (8). Previous studies investigating cancer development have examined various genes, including oncogenes, tumor suppressor genes, DNA repair genes, and genes encoding phase I and II enzymes (9-12). GSH levels have been shown to be elevated in numerous types of human cancer, including bone marrow $(13)$, breast $(14,15)$ and lung $(16,17)$. Therefore, understanding the role of GSH in the development of cancer will be a significant step towards cancer prevention and/or attenuation. The present study examined the expression levels of GSH and the enzymes involved in its biosynthesis in human colon cancer tissues and cell lines.

\section{Materials and methods}

Reagents. Anti-GCLC antibody was purchased from Thermo Fisher Scientific, Inc. (Waltham, MA, USA). Anti-GSS antibody was purchased from Abcam (Cambridge, MA, 
USA). Anti- $\beta$-actin antibody was purchased from Santa Cruz Biotechnology, Inc. (Dallas, TX, USA). All other chemicals and reagents used in the present study were of analytical grade.

Cell culture. The Caco-2, SNU-407, SNU-1033, HCT-116, and HT-29 human colon cancer cell lines were obtained from the Korean Cell Line Bank (Seoul, Korea). The FHC normal human colon cell line was purchased from the American Type Culture Collection (Rockville, MD, USA). All cells were maintained at $37^{\circ} \mathrm{C}$ in a humidified incubator containing $5 \% \mathrm{CO}_{2}$. The SNU-407, SNU-1033, HCT-116, and HT-29 cells were cultured in RPMI 1640 (Gibco Life Technologies, Grand Island, NY, USA) supplemented with $10 \%$ heat-inactivated fetal bovine serum (FBS; Gibco Life Technologies), $100 \mu \mathrm{g} / \mathrm{ml}$ streptomycin (Gibco Life Technologies) and $100 \mathrm{U} / \mathrm{ml}$ penicillin (Gibco Life Technologies). The Caco-2 cells were cultured in Minimal Essential medium (Gibco Life Technologies) supplemented with $10 \%$ heat-inactivated FBS, $100 \mu \mathrm{g} / \mathrm{ml}$ streptomycin, and $100 \mathrm{U} / \mathrm{ml}$ penicillin. The FHC cells were cultured in a 1:1 mixture of Ham's F12 and Dulbecco's modified Eagle's medium (Gibco Life Technologies) supplemented with $25 \mathrm{nM}$ HEPES (Gibco Life Technologies), $10 \mathrm{ng} / \mathrm{ml}$ cholera toxin (EMD Millipore, Billerica, MA, USA), $5 \mu \mathrm{g} / \mathrm{ml}$ insulin (Sigma-Aldrich, St. Louis, MO, USA), $5 \mu \mathrm{g} / \mathrm{ml}$ transferrin (Sigma-Aldrich), 100 ng/ml hydrocortisone (Sigma-Aldrich) and $10 \%$ FBS.

Patient tissue. The colon tissue samples were obtained from 15 human patients ( 3 female, 12 male; median age 66, age range, 43-86; 1 stage I, 3 stage II and 11 stage III) with colon cancer who were treated at the Jeju National University Hospital (Jeju, Korea). Normal and cancerous sections of tissue $(\sim 0.5-1 \mathrm{~cm})$ were collected from each patient during the operation. The present study was approved by the institutional review board for ethics of the Jeju National University Hospital (IRB:2011-38), and written informed consent was obtained from the patients.

Intracellular GSH measurement. The intracellular GSH content was measured using the GSH-400 Colorimetric Assay kit (Oxis Biotech Inc., Portland, OR, USA). The cells and tissues were lysed with lysis buffer and were harvested, homogenized in a metaphosphoric working solution, and subsequently centrifuged at $15,000 \mathrm{x} \mathrm{g}$ for $10 \mathrm{~min}$. The obtained supernatant was mixed with an equal amount of trichloroacetic acid (a precipitation reagent provided by the GSH-400 assay kit) and further centrifuged at 15,000 x g for 5 min. A total of $50 \mu 1 \mathrm{R} 1$ solution (from the GSH-400 kit), supplemented with chromogenic reagent in $\mathrm{HCl}$ was added to $900 \mu \mathrm{l}$ supernatant, prior to being gently centrifuged at $15,000 \mathrm{x} g$ for $5 \mathrm{~min}$. A total of $50 \mu \mathrm{l} \mathrm{R} 2$ solution $(30 \% \mathrm{NaOH}$; from the GSH-400 kit) was then added to the solution, and the mixtures were incubated at $25 \pm 3^{\circ} \mathrm{C}$ for $10 \mathrm{~min}$. Following centrifugation at $15,000 \mathrm{x}$ g for $5 \mathrm{~min}$, the absorbance of the clear supernatant was measured at $400 \mathrm{~nm}$ using a Scanning Multi-Well Spectrophotometer (Sunrise; Tecan Group, Ltd., Salzburg, Austria).

RNA isolation and reverse transcription-quantitative polymerase chain reaction $(R T-q P C R)$. Total RNA was isolated from the cells using the easy-BLUE ${ }^{\mathrm{TM}}$ Total RNA Extraction kit (Intron Biotechnology Inc., Seongnam, Korea) and template RNA was amplified using $1 \mu \mathrm{l}$ RT reaction buffer, along with primers (Bioneer Corporation, Daejeon, Korea), dNTPs, and 0.5 U Taq DNA polymerase (Invitrogen Life Technologies, Carlsbad, CA, USA), in a final volume of $25 \mu \mathrm{l}$. The PCR conditions were as follows: Initial denaturation at $94^{\circ} \mathrm{C}$ for $5 \mathrm{~min}$; followed by 35 cycles of $94^{\circ} \mathrm{C}$ for $30 \mathrm{sec}, 60^{\circ} \mathrm{C}$ for $30 \mathrm{sec}, 72^{\circ} \mathrm{C}$ for $30 \mathrm{sec}$, and a final elongation step at $72^{\circ} \mathrm{C}$ for $7 \mathrm{~min}$. The following primers were used to amplify the GCLC and GSS cDNA: GCLC forward, 5'-AGTTCAATACAGTTGAGG-3', reverse, 5'-TACTGATCCTATAGTTAT-3' (350 bp); GSS forward, 5'-CTGGAGCGGCTGAAGGACA-3', reverse, 5'-AGCTCTGAGATGCACTGGACA-3' (806 bp); and GAPDH forward, 5'-GTGGGCCGCCCTAGGCACCAGG-3'; and reverse, 5'-GGAGGAAGAGGATGCGGCAGTG-3' (868 bp). Amplifications were performed on a MyCycler Thermocycler (Bio-Rad Laboratories, Inc., Hercules, CA, USA). The amplified products were separated by electrophoresis on a $1 \%$ agarose gel, and stained with RedSafe ${ }^{\mathrm{TM}}$ nucleic acid staining solution (Intron Biotechnology Inc.), prior to gel image capture under UV light using Image Quant ${ }^{\mathrm{TM}} \mathrm{TL}$ analysis software (GE Healthcare Life Sciences, Chalfont, UK).

Immunoblot analysis. The cells were lysed on ice for $30 \mathrm{~min}$ in $100 \mu 1$ lysis buffer, $(120 \mathrm{mM} \mathrm{NaCl}, 40 \mathrm{mM}$ Tris $\mathrm{pH} 8.0$, and $0.1 \% \mathrm{NP} 40$ ) prior to being centrifuged at $13,000 \mathrm{x} \mathrm{g}$ for $15 \mathrm{~min}$. The supernatants were collected and the protein concentration was determined using the Bradford method (Bio-Rad Laboratories, Inc.). Aliquots containing $40 \mu \mathrm{g}$ protein were boiled for $5 \mathrm{~min}$ prior to being separated by $10 \%$ SDS-PAGE. The proteins were then transferred onto nitrocellulose membranes (EMD Millipore) and the blots were blocked with $1 \%$ bovine serum albumin (Sigma-Aldrich) in Tris-buffered saline with $1 \%$ Tween-20 for $1 \mathrm{~h}$. The blots were then incubated with GCLC (PA5-19702; 1:1,000), GSS (ab91591; 1:1,000), or $\beta$-actin (sc-47778; 1:2,000) antibodies at $4^{\circ} \mathrm{C}$ overnight. The membranes were subsequently incubated with horseradish peroxidase-conjugated secondary immunoglobulin G antibodies (Pierce Biotechnology Inc., Rockford, IL, USA). The protein bands were detected using an Enhanced Chemiluminescence Western Blotting Detection kit (GE Healthcare Life Sciences). Blots were quantified using ImageJ software, version 1.47 (National Institutes of Health, Bethesda, MD, USA)

Immunohistochemistry. The colon tissue specimens were fixed in $10 \%$ buffered formalin and embedded in paraffin. The tissue blocks were cut into $3 \mu \mathrm{m}$ sections and mounted onto Superfrost Plus-coated slides (Thermo Fisher Scientific, Inc.). The sections were then deparaffinized in xylene and rehydrated through a series of graded ethanol: 2 min in 100\% twice, 2 min in $95 \%, 2 \mathrm{~min}$ in $70 \%$ and $2 \mathrm{~min}$ in $50 \%$. The staining was performed using a BenchMark XT Immunostainer (Ventana Medical Systems, Inc., Tucson, AZ, USA), according to the manufacturer's instructions. Antigen retrieval was carried out on the immunostainer at $100^{\circ} \mathrm{C}$ in EDTA buffer for $30 \mathrm{~min}$. The slides were subsequently incubated with anti-GCLC 


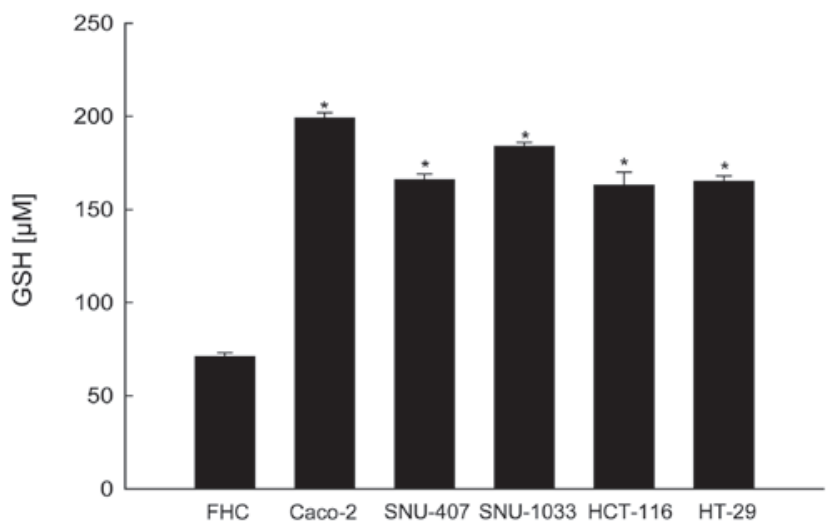

Figure 1. GSH expression levels in FHC normal colon, and Caco-2, SNU-407, SNU-1033, HCT-116 and HT-29 colon cancer cell lines. The GSH levels were measured using a colorimetric assay kit. "P<0.05, vs. FHC cells as determined by analysis of variance and Tukey's tests. GSH, glutathione.

A
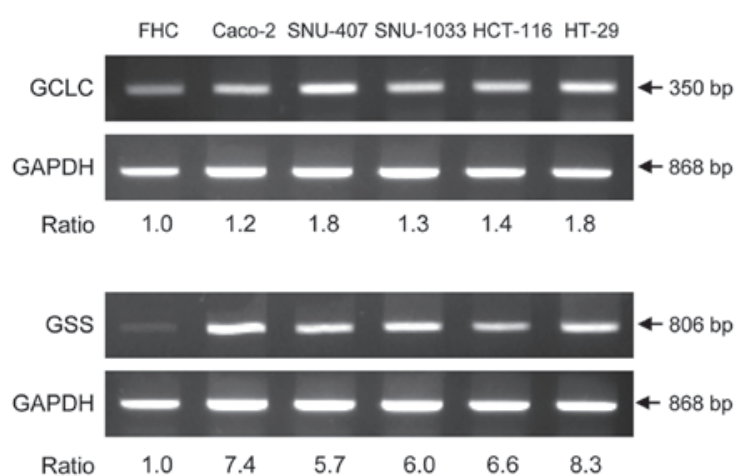

$\mathbf{B}$

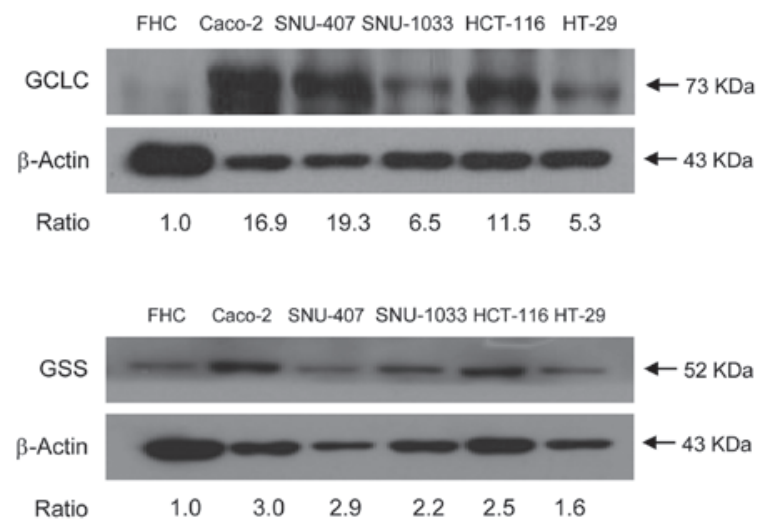

Figure 2. Expression levels of GSH metabolizing enzymes in FHC normal colon, and Caco-2, SNU-407, SNU-1033, HCT-116 and HT-29 cancer cell lines. (A) RT-qPCR analyses of the mRNAs encoding GCLC and GSS. The mRNA expression levels of GAPDH were measured as a loading control. The ratios indicate the expression levels in each cancer cell line relative to those in the normal FHC cell line. (B) Immunoblot analyses of GCLC and GSS proteins. The expression levels of $\beta$-actin were measured as a loading control. The ratios indicate the expression levels in each cancer cell line relative to those in the normal FHC cell line. GSH, glutathione; GCLC, glutamate cysteine ligase catalytic subunit; GSS, GSH synthetase; RT-qPCR, reverse transcription-quantitative polymerase chain reaction.

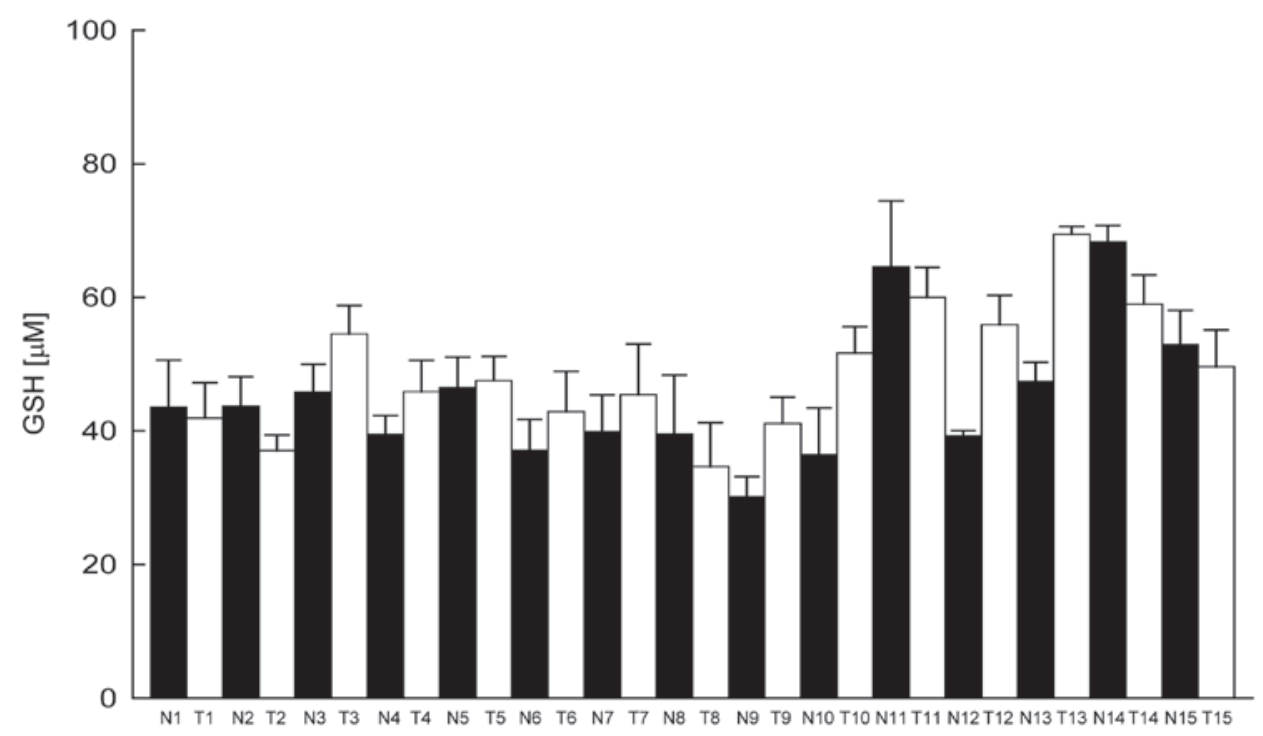

Figure 3. GSH expression levels tissue from patients with colon cancer. The GSH expression levels were measured using a colorimetric assay kit. GSH, glutathione; $\mathrm{N}$, normal; T, tumor. 
A
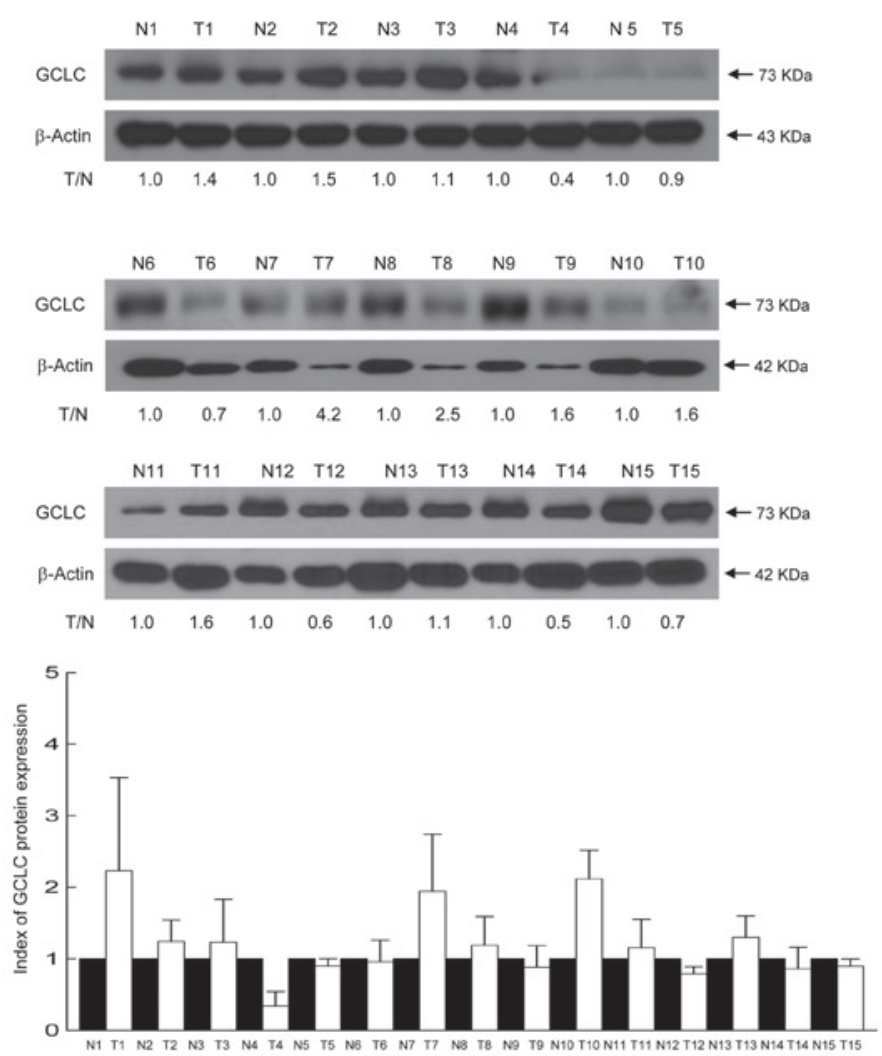

B
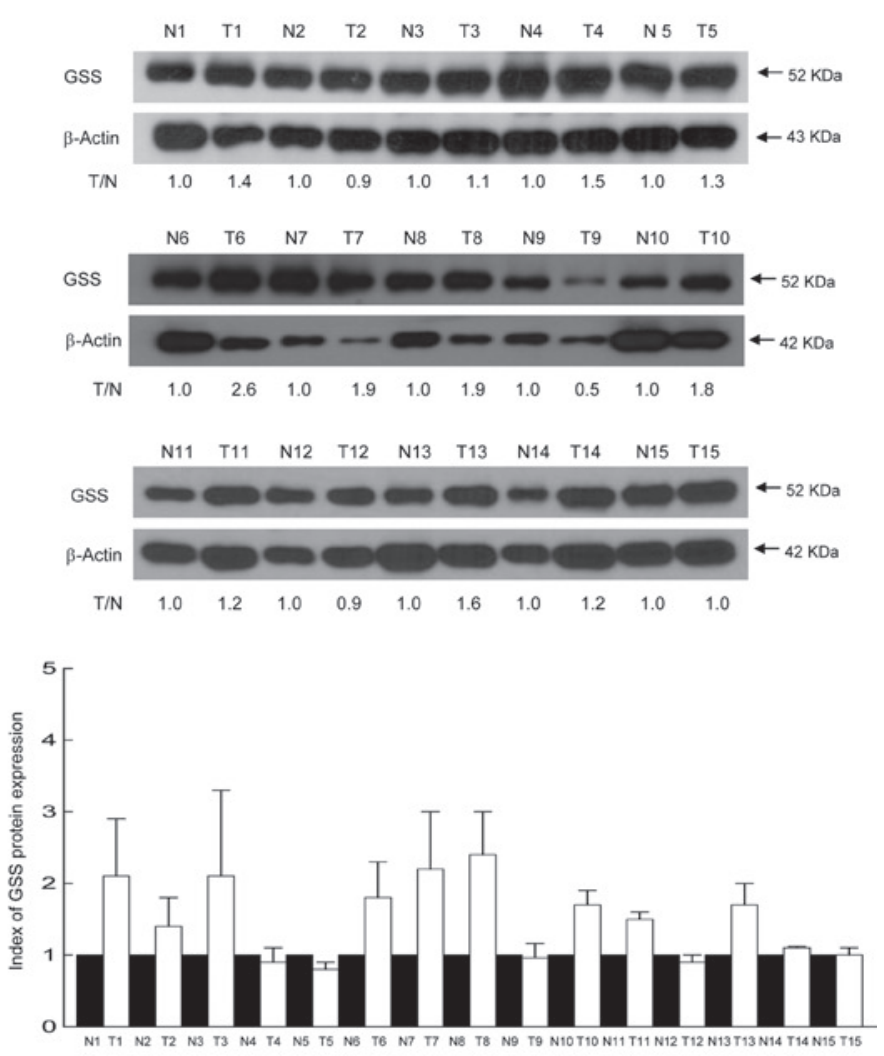

Figure 4. Expression levels of GSH synthetic enzymes in tissue from patients with colon cancer. Immunoblot analyses of (A) GCLC and (B) GSS protein expression. The expression levels of $\beta$-actin were measured as a loading control. The numbers below the blots indicate the ratio of expression in the tumor sample relative to the corresponding normal sample N, normal; T, tumor; GCLC, glutamate cysteine ligase catalytic subunit; GSS, GSH synthetase.
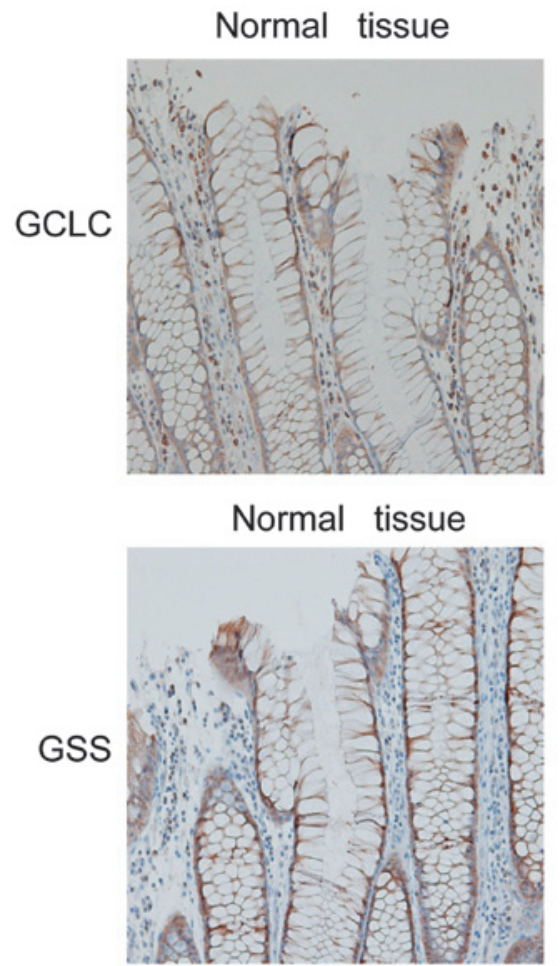

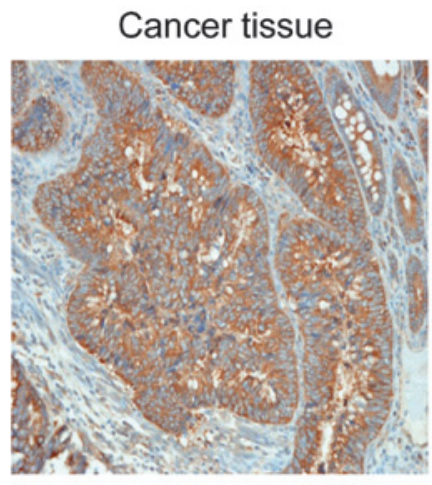

\section{Cancer tissue}

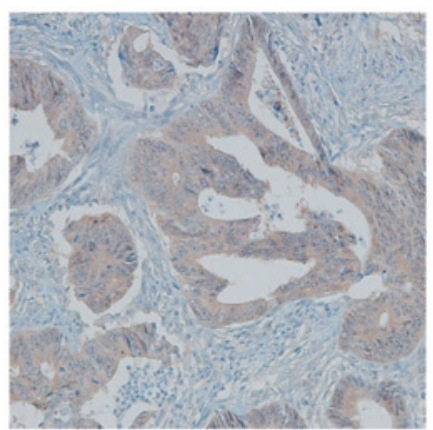

Figure 5. Immunohistochemical analyses of GCLC and GSS in colon carcinoma tissue, and in corresponding normal tissue. Magnification, x100. Low protein expression levels of GCLC and GSS are present in normal mucosa, whereas diffuse and high protein expression levels of GCLC and GSS are present in cancerous tissue. GCLC, glutamate cysteine ligase catalytic subunit; GSS, GSH synthetase. 
and anti-GSS antibodies $(1: 1,000)$ at $37^{\circ} \mathrm{C}$ for $32 \mathrm{~min}$, and 3,3'-diaminobenzidine was used as a chromogen. The slides were counterstained with hematoxylin (Sigma-Aldrich) prior to mounting, and were evaluated using a light microscope (Olympus BX51; Olympus, Center Valley, PA, USA) and interpreted by a pathologist.

Statistical analysis. Statistical significance was determined using analysis of variance and Tukey's tests with SigmaStat software, version 3.0 (Systat Software, Inc., San Jose, CA, USA). All values are presented as the mean \pm standard error. $\mathrm{P}<0.05$ was considered to indicate a statistically significant difference.

\section{Results}

Expression levels of GSH and GSH metabolizing enzymes in human colon cancer cell lines. The intracellular GSH levels were assessed in a normal human colon cell line (FHC) and five human colon cancer cell lines (Caco-2, SNU-407, SNU-1033, HCT-116, and HT-29) using a commercially available colorimetric assay kit. The intracellular GSH levels were significantly higher in all five of the colon cancer cell lines, as compared with the normal colon cell line (Fig. 1). Concordant with these results, RT-qPCR and immunoblot analyses revealed that the mRNA and protein expression levels of the GSH synthetic enzymes: GCLC and GSS, were also higher in the cancer cell lines, as compared with the normal colon cell line (Fig. 2A and 2B). Although the expression levels of GSH and GSH synthetic enzymes differed among the colon cancer cell lines, the results of the present study suggest that the expression levels of GSH and GSH metabolizing enzymes may serve as useful biomarkers for the detection of colon cancer.

Expression levels of GSH and GSH metabolizing enzymes in normal and colon carcinoma tissue samples. The expression levels of GSH were then examined in the normal and cancerous colon tissue samples of 15 patients undergoing treatment at the Jeju National University Hospital. In nine out of 15 patients, GSH expression levels were higher in the tumor tissue samples, as compared with the corresponding normal tissue samples (Fig. 3). Immunoblot analyses showed that the protein expression levels of GCLC were higher in tumor tissue samples, as compared with the corresponding normal tissue samples in $53 \%(8 / 15)$ of the patients with colon cancer (Fig. 4A). Similarly, the protein expression levels of GSS were higher in tumor tissue samples, as compared with the corresponding normal tissue samples in 67\% (10/15) of the patients (Fig. 4B). Furthermore, immunohistochemical analyses of the colon tissue sections revealed low protein expression levels of GCLC and GSS in normal mucosa, whereas diffuse and high expression levels of these proteins were detected in the cancerous tissue (Fig. 5).

\section{Discussion}

GSH is a major cellular antioxidant and is crucial for maintaining the balance between oxidation and antioxidation $(2,18)$. GSH is also important in cellular detoxification and is associated with numerous aspects of the immune response (2). Enhanced resistance to chemotherapeutic drugs and radiotherapy is associated with GSH-conjugation and detoxification (5-7). Previous studies have reported that the expression levels of GSH are elevated in numerous types of human cancer tissue, and increased expression levels of GCLC have also been identified in lung, breast, liver, and other types of cancer $(19,20)$. In addition, GCLC and GSS were highly expressed in the mammary tumors and can be used as prognostic markers for mammary neoplasm (21). Furthermore, a previous study demonstrated that drug-resistance is associated not only with elevated GSH levels, but also with increased activity of GCLC (22).

The results of the present study demonstrated that the GSH levels, and the mRNA and protein expression levels of GCLC and GSS were higher in five human colorectal cancer cell lines, as compared with normal human colon cell lines. Similar results were also observed in cancerous and normal tissue samples, which were collected from 15 patients with colon cancer. The relatively high expression levels of GCLC and GSS in colon cancer tissue, as compared with normal tissue, were confirmed by an immunohistochemical analysis.

In conclusion, the results of the present study suggest that the expression levels of GSH and GSH metabolizing enzymes may serve as clinically useful biomarkers of colon cancer, as well as potential targets for anticancer drugs. Further study is required to elucidate the mechanisms of the GSH metabolizing enzyme upregulation in human colorectal cancer cell lines and tissues.

\section{Acknowledgements}

The biospecimens and data used in the present study were provided by the Biobank of Jeju National University Hospital. The present study was supported by a grant from the National R\&D Program for Cancer Control, Ministry for Health and Welfare, Korea (grant no. 1120340).

\section{References}

1. Lu SC: Regulation of glutathione synthesis. Mol Aspects Med 30: 42-59, 2009.

2. Lu SC: Glutathione synthesis. Biochim Biophys Acta 1830: 3143-3153, 2013.

3. Huang HC, Nguyen $\mathrm{T}$ and Pickett CB: Regulation of the antioxidant response element by protein kinase $\mathrm{C}$-mediated phosphorylation of NF-E2-related factor 2. Proc Natl Acad Sci USA 97: 12475-12480, 2000.

4. Kuo MT: Redox regulation of multidrug resistance in cancer chemotherapy: Molecular mechanisms and therapeutic opportunities. Antioxid Redox Signal 11: 99-133, 2009.

5. Nguyen LN, Munshi A, Hobbs ML, Story MD and Meyn RD Jr: Paclitaxel restores radiation-induced apoptosis in a bcl-2-expressing, radiation-resistant lymphoma cell line. Int J Radiat Oncol Biol Phys 49: 1127-1132, 2001.

6. Vukovic V, Nicklee T and Hedley DW: Microregional heterogeneity of non-protein thiols in cervical carcinomas assessed by combined use of HPLC and fluorescence image analysis. Clin Cancer Res 6: 1826-1832, 2000.

7. Vukovic V, Nicklee T and Hedley DW: Differential effects of buthionine sulphoximine in hypoxic and non-hypoxic regions of human cervical carcinoma xenografts. Radiother Oncol 60: 69-73, 2001.

8. Herceg Z: Epigenetics and cancer: Towards an evaluation of the impact of environmental and dietary factors. Mutagenesis 22: 91-103, 2007.

9. Arvelo F, Sojo F and Cotte C: Biology of colorectal cancer. Ecancermedicalscience 9: 520, 2015. 
10. Singh S: Cytoprotective and regulatory functions of glutathione S-transferases in cancer cell proliferation and cell death. Cancer Chemother Pharmacol 75: 1-15, 2015.

11. Dizdaroglu M: Oxidatively induced DNA damage and its repair in cancer. Mutat Res Rev Mutat Res 763: 212-245, 2015.

12. Laborde E: Glutathione transferases as mediators of signaling pathways involved in cell proliferation and cell death. Cell Death Differ 17: 1373-1380, 2010

13. Joncourt F, Oberli-Schrämmli AE, Stadler M, Buser K, Franscini L, Fey MF and Cerny T: Patterns of drug resistance parameters in adult leukemia. Leuk Lymphoma 17: 101-109, 1995.

14. Perry RR, Mazetta JA, Levin M and Barranco SC: Glutathione levels and variability in breast tumors and normal tissue. Cancer 72: 783-787, 1993.

15. Jardim BV, Moschetta MG, Leonel C, Gelaleti GB, Regiani VR, Ferreira LC,Lopes JR and Zuccari DA: Glutathione and glutathione peroxidase expression in breast cancer: An immunohistochemical and molecular study. Oncol Rep 30: 1119-1128, 2013.

16. Cook JA, Pass HI, Iype SN, Friedman N, DeGraff W, Russo A and Mitchell JB: Cellular glutathione and thiol measurements from surgically resected human lung tumor and normal lung tissue. Cancer Res 51: 4287-4294, 1991.
17. Oberli-Schrämmli AE, Joncourt F, Stadler M, et al: Parallel assessment of glutathione-based detoxifying enzymes, O6-alkylguanine-DNA alkyltransferase and P-glycoprotein as indicators of drug resistance in tumor and normal lung of patients with lung cancer. Int J Cancer 59: 629-636, 1994.

18. Sies H: Glutathione and its role in cellular functions. Free Radic Biol Med 27: 916-921, 1999.

19. Soini Y, Karihtala P, Mäntyniemi A, Turunen N, Pääkko P and Kinnula V: Glutamate-L-cysteine ligase in breast carcinomas. Histopathology 44: 129-135, 2004.

20. Mougiakakos D, Okita R, Ando T, et al: High expression of GCLC is associated with malignant melanoma of low oxidative phenotype and predicts a better prognosis. J Mol Med (Berl) 90: 935-944, 2012.

21. Leonel C, Gelaleti GB, Jardim BV, et al: Expression of glutathione, glutathione peroxidase and glutathione $\mathrm{S}$-transferase pi in canine mammary tumors. BMC Vet Res 10: 49, 2014.

22. Bailey HH, Gipp JJ, Ripple M, Wilding G and Mulcahy RT: Increase in gamma-glutamylcysteine synthetase activity and steady-state messenger RNA levels in melphalan-resistant DU-145 human prostate carcinoma cells expressing elevated glutathione levels. Cancer Res 52: 5115-5118, 1992. 